\title{
Analysis of Context-Aware based Traffic Flow Optimization Algorithm across the Integrated Heterogeneous Wireless Networks Using Multi-MAC Switching Management Scheme
}

\author{
Jinho Lee, Ronnie D. Caytiles, and Byungjoo Park* \\ Department of Multimedia Engineering, Hannam University \\ 133 Ojeong-dong, Daeduk-gu, Daejeon, Korea \\ jin@iitels.com,rdcaytiles@gmail.com, "bjpark@hnu.kr
}

\begin{abstract}
Wireless mesh networks evolved from a simple ad hoc network to complex integration of different heterogeneous wireless networks. It includes a variety of telecommunication standards wherein cooperation and interoperability is essentially important. This paper analyses the flow distribution management of multimedia contents over a multi-MAC integrated heterogeneous wireless networks. The host-based mobility management protocols are constrained with signaling overheads, higher handover latency, and higher packet loss rate. This paper deals with the analysis of the interoperability among multiple MACs in order to efficiently manage the flow distribution of multimedia contents across the integrated heterogeneous wireless networks. The mobile device within the coverage area of multiple radio access networks or in a heterogeneous environment will have the capability to choose among connections to the best available network. The network-based mobility management of PMIPv6 has been utilized to optimize the flow mobility distribution of multimedia contents.
\end{abstract}

Keywords: Multi-MAC, flow mobility distribution, PMIPv6, network-based mobility management

\section{Introduction}

Nowadays, it is very rare to see an isolated network. Almost every network is connected and is able to access the multimedia contents over the Internet. The complex integration of these heterogeneous wireless networks had led to the increasing demand for seamless and balanced flow mobility for the distribution of multimedia contents. The continuous network connectivity in the integrated heterogeneous wireless networks of user's mobile devices moving from one point of attachment (PoA) to the next can be delivered by a robust mobility management scheme. The seamless flow distribution of multimedia contents can be warranted through a robust mobility management allowing the heterogeneous wireless networks to cooperate and become integrated.

The increasing number of mobile users and the amassed volume of multimedia contents in the Internet require a seamless real-time communication session. It demands for a seamless and efficient flow mobility distribution of multimedia contents. The hostbased mobility management protocols being implemented to provide mobility support suffers from numerous drawback such as higher handover latency (due to a number of signaling that a mobile device is involved), packet losses (due to the handover delays and disruptions caused by the exchanges of registration signaling, and routability issues), and signaling overhead (the mobile device needs to update its movement to the network every time it moves). In addition, mobile devices are generally resource-constrained, that is,

${ }^{*}$ Corresponding Author 
power consumption, processing power, memory, etc. The amount of signaling that these mobile devices are involved causes them to waste their resources.

Thus, the network-based mobility management protocol for supporting the mobility of the mobile devices in a heterogeneous wireless networks can be of more efficient performance. That is since the mobility-related signaling was relegated to the network instead of requiring it to the mobile device. This allows the mobile device to move freely between different access networks without the burden of registering its address in every movement.

This paper aims to provide an analysis of a multi-MAC flow mobility distribution of multimedia contents across the integrated heterogeneous wireless networks. The analysis is based on a network-based mobility management of PMIPv6 protocol that allows the user's mobile devices (i.e., mobile node or $\mathrm{MN}$ ) to roam across different wireless networks without being required of any signaling to register its movements. The proposed scheme can be able to determine the optimized path for the flow mobility distribution of multimedia contents that is based on the available traffic conditions among the various wireless networks. The seamless connectivity and uninterrupted multimedia contents distribution is guaranteed through an efficient handoff scheme over the integrated heterogeneous wireless networks.

The rest of this paper is organized as follows: Section 2 discusses optimization techniques for the enhancement of MIPv6 and their corresponding operations; the analysis for a multi-MAC flow mobility distribution of multimedia contents across the integrated heterogeneous wireless networks is outlined in Section 3; and the concluding remarks in Section 4.

\section{Optimizations for MIPv6}

The complexity of integrated heterogeneous wireless networks faces the challenges of establishing backhaul deployment and technologies, inter-cell interference coordination and management, and the mobility management. The issue of mobility management is essentially important for achieving the optimized coverage and capacity of these integrated heterogeneous wireless networks. The changes in the point of attachment (PoA) of every user's mobile device can be tracked and on-going connections can be maintained through a seamless and optimized mobility management. This section presents both the host-based optimizations for the standard mobile IPv6 and the corresponding operations.

The mobile node (MN) in the host-based mobility management is required to participate in the signaling related to its movement across the integrated heterogeneous wireless networks (i.e., changing the MN's point of attachment or PoA). The host-based mobility management affects the entire protocol stack, that is, the $\mathrm{MN}$ is required for protocol stack modification. That includes from the physical layer, data link, and network layers up to the transport and application layers. The $\mathrm{MN}$ is required to participate in the mobility signaling when roaming across different network domains and support its mobility. The mobility management signaling wherein the $\mathrm{MN}$ is involved includes movement detection (MD) messages, Router Solicitation (RtSol) requests, Duplicate Address Detection (DAD), Binding Update (BU) messages, etc.

The Internet Engineering Task Force (IRTF) have standardized the MIPv6 to address the drawbacks for MIPv4 wherein the continuously increasing number of mobile users are suffering from high signaling loads, depreciating number of addresses, packet delays due to triangular routing, and other issues $[1,2,4]$. MIPv6 is a global mobility support that enables the $\mathrm{MN}$ to maintain its on-going connection with its $\mathrm{CN}$ while moving within the Internet domain. The $\mathrm{CN}$ sends the multimedia contents IP packets to the MN through the HA. The MN is provided with a fixed home address (HoA) by the HA and a temporary care-of address ( $\mathrm{CoA})$ whenever it visits another network. When the MN is away with its 
home network, the CoA is used since the $\mathrm{MN}$ will not be reachable through its HoA. Thus, the IP packets that are sent by the CN which are addressed to the MN's HoA will be intercepted by the HA. These IP packets will be tunneled by the HA to the MN's current CoA.

The MN needs to update the HA whenever it moves between access networks in order to update its current CoA by sending binding update (BU) messages. The HA then maps the MN's home address with its corresponding CoA in its binding cache. The tunneling of IP packets ends at the MN directly, unlike with the MIPv4 where it only ends with the foreign agent (FA). The MIPv6 also introduced a route optimization in order to address the triangular routing problem. Route optimization enables the $\mathrm{MN}$ and $\mathrm{CN}$ to communicate directly without the intervention of the HA. That is, the MN needs to send binding update $(\mathrm{BU})$ message to the $\mathrm{CN}$ (similar process with registration to the $\mathrm{HA}$ ) in order that the $\mathrm{CN}$ is updated with the MN's current location. This improves the network performance through reliability, security, and reduced network loads.

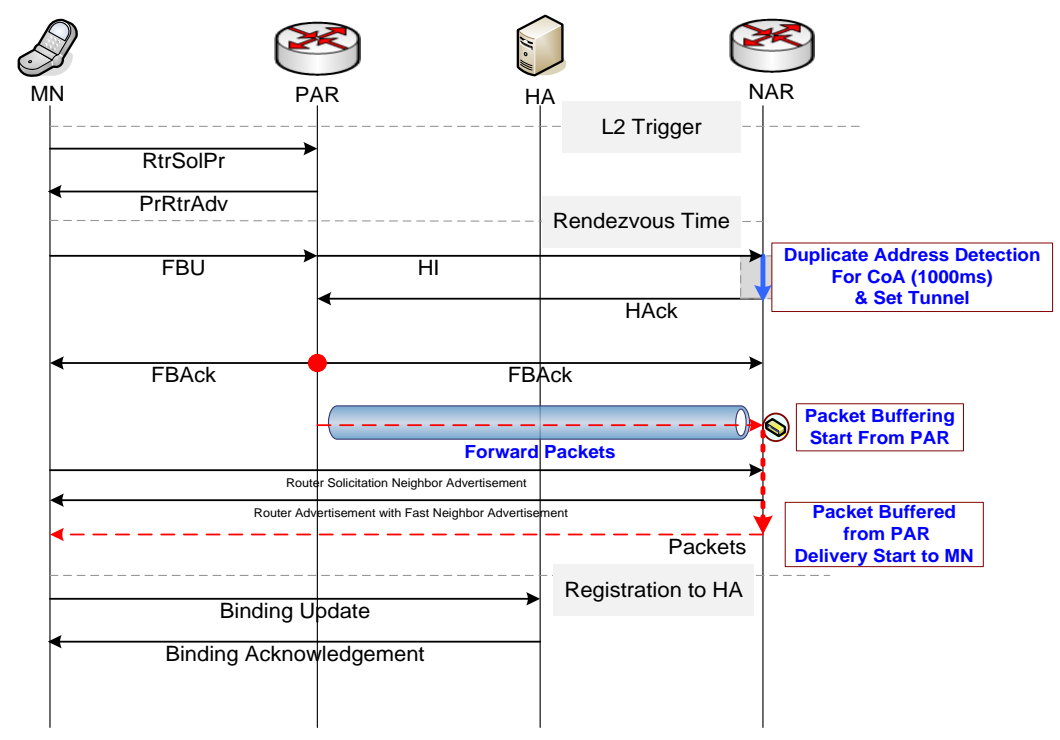

(a) FMIPv6 Operations

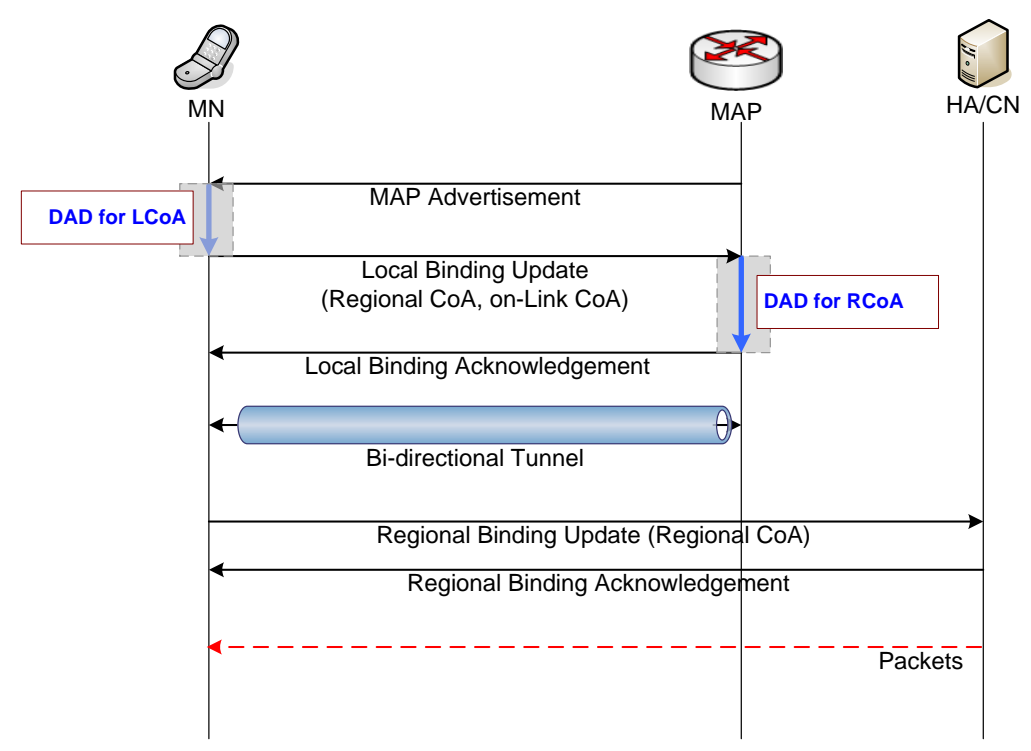

(b) Hierarchical MIPv6 Operations

Figure 1. Host-based Mobility Management Optimizations 
The Fast MIPv6 (FMIPv6) was proposed in order to address the handover related issues of MIPv6 [3, 4]. The handover performance is improved by using link layer trigger events. The MN's movement between access networks is anticipated in order to minimize the service interruption and packet loss. The functional operations of FMIPv6 are depicted in Figure 1(a). The MN's movement is anticipated by sending a router solicitation for proxy advertisement (RtSolPr) message to the previous AR in order to request for a CoA. A proxy router advertisement (PrRtAdv) message will then be replied by the previous AR containing a new CoA to be used by the MN on the new AR's link. The fast handover will be initiated by the MN through sending a fast binding update (FBU) message containing the obtained new CoA to the previous AR. This message is sent in order to inform the previous AR to start forwarding the IP packets the new CoA located at the new AR's link. To realize this, the previous AR needs to establish a tunnel to the new AR. Before the tunnel is to be set, an address duplication check will be performed at the new AR after the previous AR has sent a handover initiate (HI) message. A handover acknowledge (HAck) message is replied by the new AR whenever there is no address duplication and the tunnel is successfully established. The previous AR sends a fast binding acknowledgment (FBack) to the MN and the new AR as soon as it received the HAck message. The IP packets can now be forwarded and back from the previous AR to the new AR.

The Hierarchical Mobile Internet Protocol version 6 (HMIPv6) refers to a local mobility management protocol that allows a frequently moving $\mathrm{MN}$ to have a locally managed handoffs $[5,6]$. It is primarily designed to limit the handover latency and signaling overheads and thus improving the handoff speed for mobile connection services. HMIPv6 introduces a new network entity refers to as the mobility anchor point (MAP) that serves as the focal point for the MN's seamless mobility. Figure 1(b) depicts the functional operations HMIPv6. The MN that is roaming around the HMIPv6 domain discovers the global address of the MAP from router advertisement (RtrAdv) messages sent by available access routers (ARs) within the control of the MAP. The MN can determine whether it is still located within the same MAP domain or it has moved to another MAP if the global address that it receives from RtrAdv messages is different.

The MN that enters the HMIPv6 domain is provided with two addresses:

- Regional Care-of Address (RCoA) is an address that is based on the interface identifier of the MN and the subnet prefix of the MAP. It indicates the location of the MAP to which the AR is attached.

- On-link Care-of Address (LCoA) is an address that is based on the MN's interface identifier and the subnet prefix of the default access router to which it is currently attached. It identifies the current location of the MN.

When the RCoA and LCoA were obtained by the MN, it send a BU message to the MAP containing the RCoA in its Home Address option and uses the LCoA as the source address. The MAP replies with a BA message as soon as the binding of the MN's CoA to its RCoA is successful. A bi-directional tunnel will then be established between the MAP and the MN. The multimedia contents IP packets sent by the $\mathrm{CN}$ addressed to the $\mathrm{MN}$ will be intercepted by the MAP and tunnels them directly to the MN through the LCoA. In a similar way, the IP packets sent by the MN are tunneled to the MAP which is addressed by the RCoA.

If the $\mathrm{MN}$ moves from the previous $\mathrm{AR}$ to the new $\mathrm{AR}$, the $\mathrm{MN}$ only needs to update the MAP by sending a local BU message with its new LCoA (i.e., based on the subnet prefix of the new AR). The bi-directional tunnel will then be transferred between the MAP and the new AR to where the MN is currently attached. The MAP acts as the home agent (HA) for the MN as well as the central controller for the available ARs within its domain.

However, if the MN moves to another MAP domain, the MN needs to configure a new RCoA and LCoA. The MN then needs to update the new MAP with a local BU that 
includes its new LCoA, and a home BU will be sent to the HA in order to update its new RCoA. Finally, all packets that are addressed to the MN can then be intercepted by the new MAP and tunneled to the MN's new LCoA.

In summary, the standard MIPv6 needs to update its new PoA to the HA and CN whenever it moves from one access network to another. Thereby, the implementation of MIPv6 raises several issues such as high handover latency, high packet loss, and signaling overheads. The FMIPv6 handover optimization was generally based on an accurate handover prediction and movement anticipation of the MN. The false prediction will lead to erroneous handover detection and will undesirably affect the handover's seamlessness. Likewise, the handling of the local mobility for HMIPv6 could have an advantage over the standard MIPv6; however, longer handover latency is possible if the MAPs are topologically far.

In general, the $\mathrm{MN}$ in host-based mobility management optimizations is involved in all mobility-related signaling in order to have mobility support. The protocol stack of the MN needs modification and its IP addresses changes in order to support its mobility across heterogeneous wireless networks. The MN's involvement with the mobility-related signaling can increase the MN's complexity, power consumption, and radio resources. Thus, The QoS requirements for real-time and multimedia services will not be addressed fully by host-based mobility management protocols for its drawbacks remains (e.g., higher handover latency, higher packet loss rate, and signaling overhead).

\section{Network-based Mobility Management Optimization}

The network entities on the network-based mobility management are the ones that process the mobility-related signaling of mobile nodes (MNs). This provides the MNs with mobility support without being required to participate in mobility management. The network-based mobility management aims to improve the performance of the host-based mobility management schemes in order to realize the quality of service (QoS) real-time services for $\mathrm{MN}$ communications.

The IETF Network-based Localized Mobility Management (NETLMM) working group has standardized the Proxy MIPv6 (PMIPv6) as an essential protocol based on the signaling concepts and functions of the standard MIPv6 [7, 8]. It allows the MNs to roam across various networks without being required with signaling and mobility management for these functionalities is relegated to the network components [8]. The basic operations of PMIPv6 are depicted in Figure 2. 


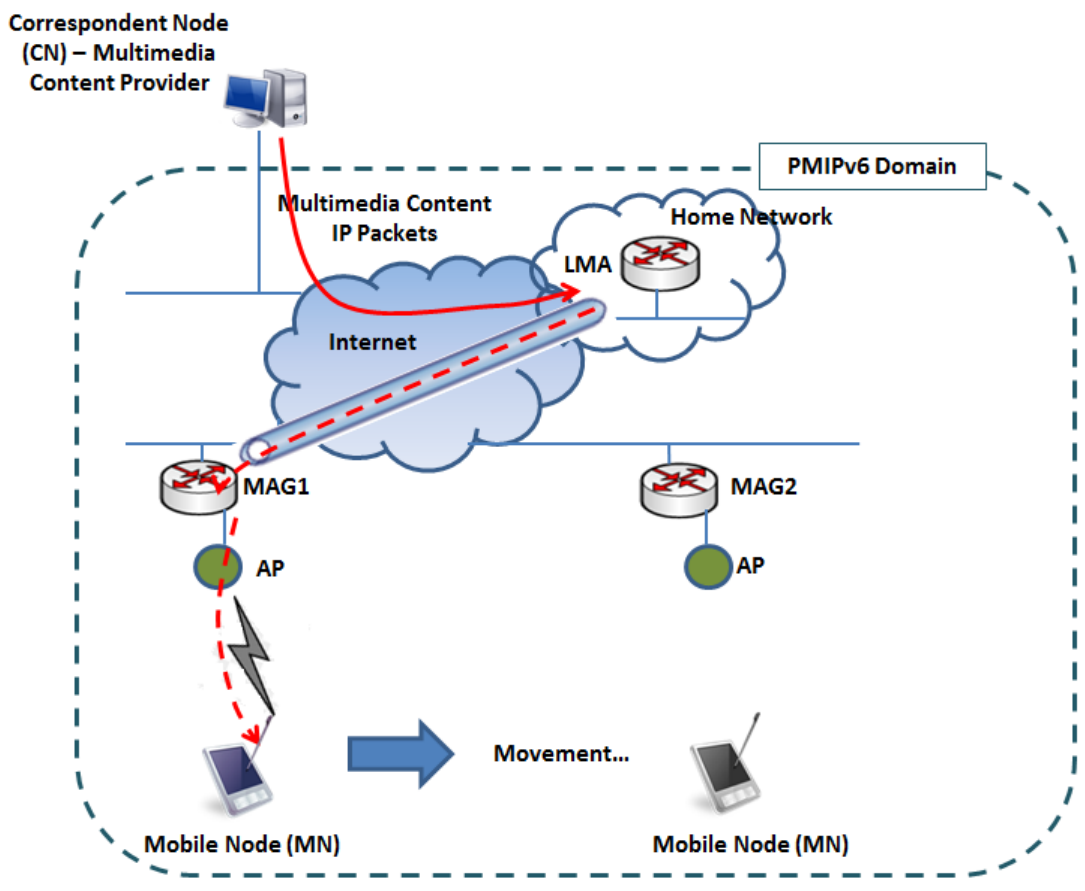

Figure 2. PMIPv6 Basic Operations

In PMIPv6, whenever an MN moves from one network to the next, the initialization of mobile signaling is performed by the proxy mobility agent within the network. The MN does not handle the exchange of signaling between the home agent (HA) and itself. A new functional entity referred to as the mobile access gateway (MAG) performs the mobilityrelated signaling such as movement detection and signaling operations on behalf of the MNs that are currently attached to its access links. The MAG usually resides in the access router (AR) wherein it detects the MN's movement, coordinates routing state updates, and provides IP connectivity with the MN.

The collection of IP address of all MNs attached within the localized mobility domain (LMD) are maintained and anchored to the network entity referred to as the local mobility anchor (LMA). The LMA, which is located within the home network, is acting as the local home agent for the corresponding LMD. A bidirectional tunnel is established between the LMA and MAG wherein regardless of the MN's location with the LMD, the assigned address for the MN is kept. The traffic flow is controlled by the LMA, thus, the multimedia contents IP packets to be delivered by the correspondent node $(\mathrm{CN})$ and are addressed to the MN can be intercepted by the LMA. These multimedia contents IP packets are then encapsulated and forwarded to the appropriate MAG via the bidirectional tunnel that was created. The MAG de-capsulate the received multimedia contents IP packets and deliver them locally to the MN, which is unmindful to its own mobility. 


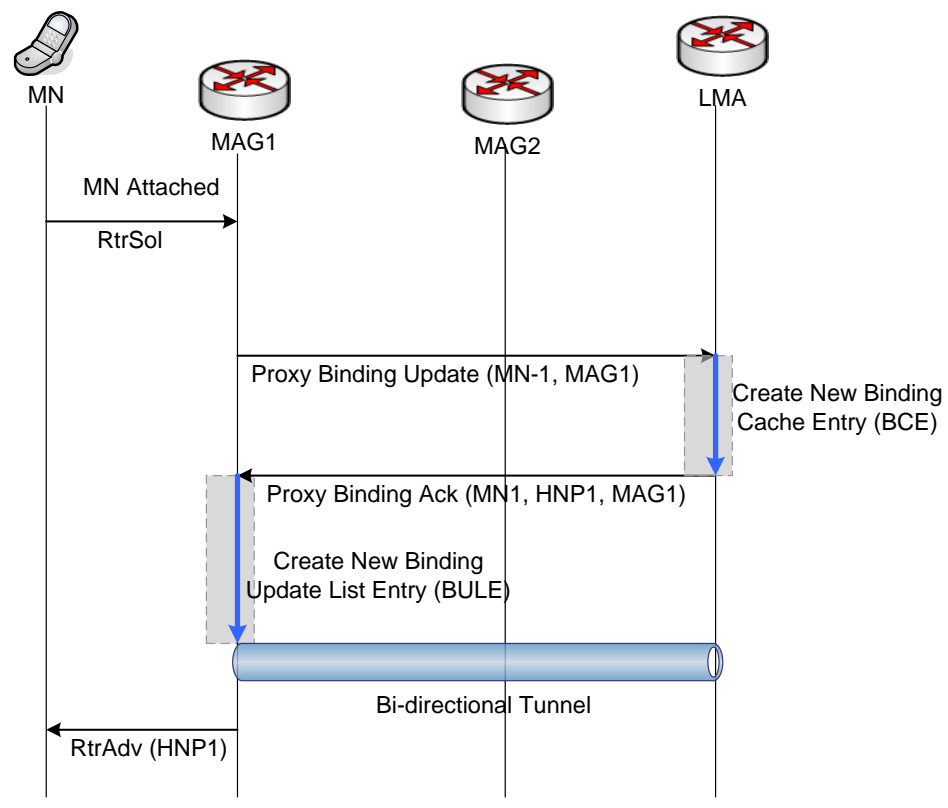

(a) Attachment of a Mobile Host to Local Mobility Anchor in PMIPv6 Domain

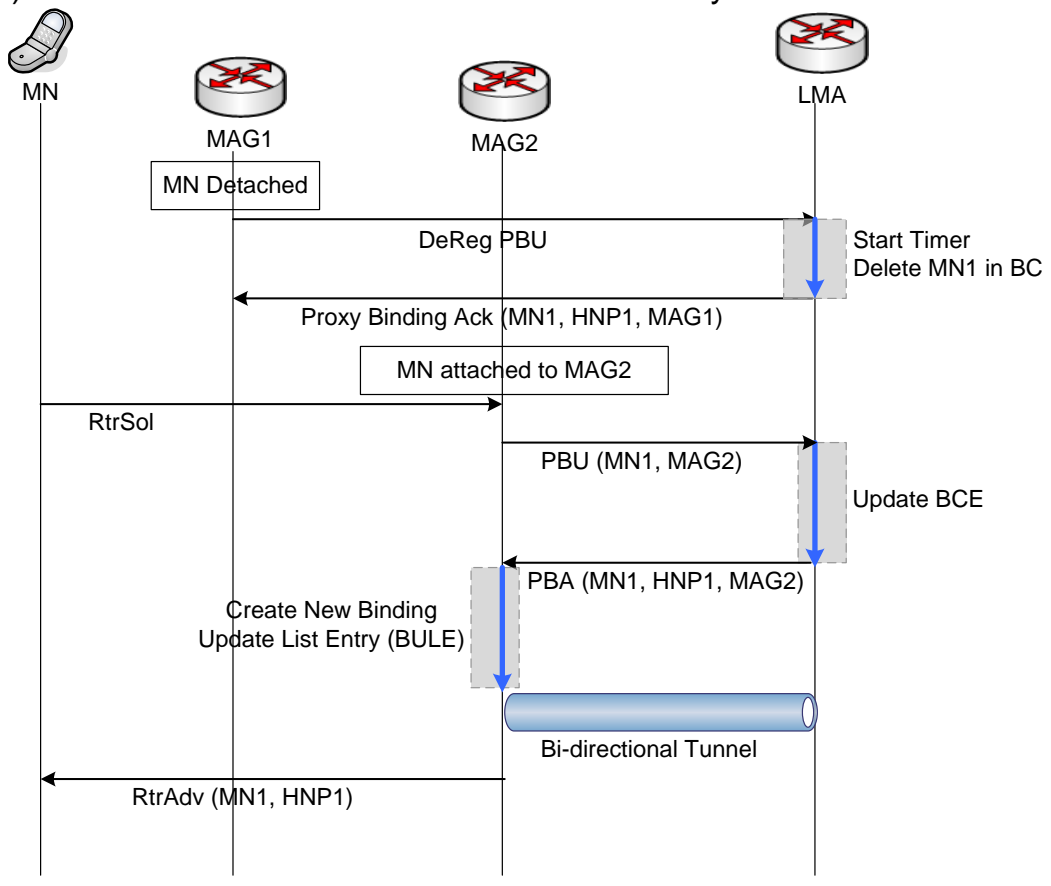

(b) A Mobile Host Changes its Attachment in PMIPv6 Domain

Figure 3. PMIPv6 Handover Operations

The overall signaling flow of PMIPv6 is shown in Figure 3. The MN that establishes its point of attachment into a PMIPv6 domain (i.e., initial attachment) is depicted in Figure 3(a). Initially, the MN sends a router solicitation (RtrSol) message as soon as it attaches to the MAG. The MAG then sends a proxy binding update (PBU) message to the LMA and in return, the LMA replies with a proxy binding acknowledgement (PBA) message with an address assigned to the MN. A new binding cache entry (BCE) is created in the LMA since the MN enters the LMD for the first time. The binding cache entry is the similar with the one maintained by the HA in the standard MIPv6. In a similar way, the MAG also maintains a binding update list entry (BULE) to keep track of all MNs that attaches and disconnects to its domain. The LMA then establishes a bidirectional tunnel 
with the MAG enabling the MN to communicate with its corresponding nodes (CNs). The initial attachment process is completed when the MAG sends a router advertisement (RtrAdv) message to the MN containing the home network prefix(es) for its address autoconfiguration.

Figure 3(b) depicts the movement of MN from the initial MAG to a new MAG. The initial MAG sends a deregistration PBU (DeReg PBU) message to the LMA whenever it detects MN's movement away from its domain and enters a new MAG. The PBU lifetime for this registration message will be zero. The LMA then accepts this request after identifying the corresponding mobility sessions for the MAG to which the request was received. Then, the LMA waits for the new MAG to send its PBU message upon detecting the presence of the MN to its access link. The LMA updates its binding cache and sends a PBA message back to the new MAG and also updates its binding update list. A new bidirectional tunnel will then be established by the LMA to the new MAG. Finally, the new MAG sends a router advertisement (RtrAdv) message that contains the MN's home network prefix(es). All multimedia contents IP packets sent from the MN are transmitted to the LMA via the bidirectional tunnel between the MAG and the LMA. On the contrary, The IP packets from the CN addressed to the MN will be intercepted by the LMA and tunneled to the MAG. The MAG then de-capsulate the IP packets and forward them to the MN.

The PMIPv6 can be considered to be relatively faster than the standard MIPv6 based on the shortened signaling update time and reduced disruption period for the MN. In addition, the network is responsible for movement tracking and mobility signaling initializations of the MN unlike with the standard MIPv6. Thus, the PMIPv6 served as the basis for the interworking of heterogeneous wireless networks. Several access technologies such as 3GPP, 3GPP2, WiMAX, and WLAN have been utilizing the PMIPv6 protocol for their mobility management allowing the access technology become independent of mobile core networks.

\section{Multi-MAC Flow Distribution Management}

This section presents a discussion of the analysis of the multi-MAC flow distribution management of multimedia contents across an integrated heterogeneous wireless networks. The seamless handover of MNs that enters the domain of the various access networks will take advantage of the features of PMIPv6 to have a network-based mobility support. In Figure 4, the integrated access networks were shown such as WiMAX, 3GPP, and WLAN. This means that, whenever the MN switches from one access network to the other, it needs to connect to a different media access controller (MAC) interface. The $\mathrm{MN}_{2}, \mathrm{MN}_{3}$, and $\mathrm{MN}_{4}$ in Figure 1 directly connect to WiMAX, 3GPP, and WLAN base stations, respectively. These mobile devices enjoy the full capacity and service of their corresponding access network. However, the major challenge is with the $\mathrm{MN}_{1}$ which moves to a location within the overlapping domains of the different access networks. That is, $\mathrm{MN}_{1}$ needs the mobility support that could efficiently manage the multi-MAC flow distribution of multimedia contents across these overlapping integrated access networks.

The $\mathrm{MN}_{1}$ needs to determine the optimized access network for sending or receiving multimedia contents IP packets from its $\mathrm{CN}$. It has the luxury of choosing to which access network offers the best service to which is required to serve its needs. To do this, the $\mathrm{MN}_{1}$ needs to identify the various network conditions to each of the available access networks such as bandwidth, number of connected MNs, volume of data traffic processed, distance of $\mathrm{MN}_{1}$ to its strongest signal area, etc. Then, depending on these factors and the $\mathrm{MN}_{1}$ user's needs, it can switch from one access network to another. The MN's mobility across these integrated heterogeneous access networks is supported by the PMIPv6 mobility management protocol. 


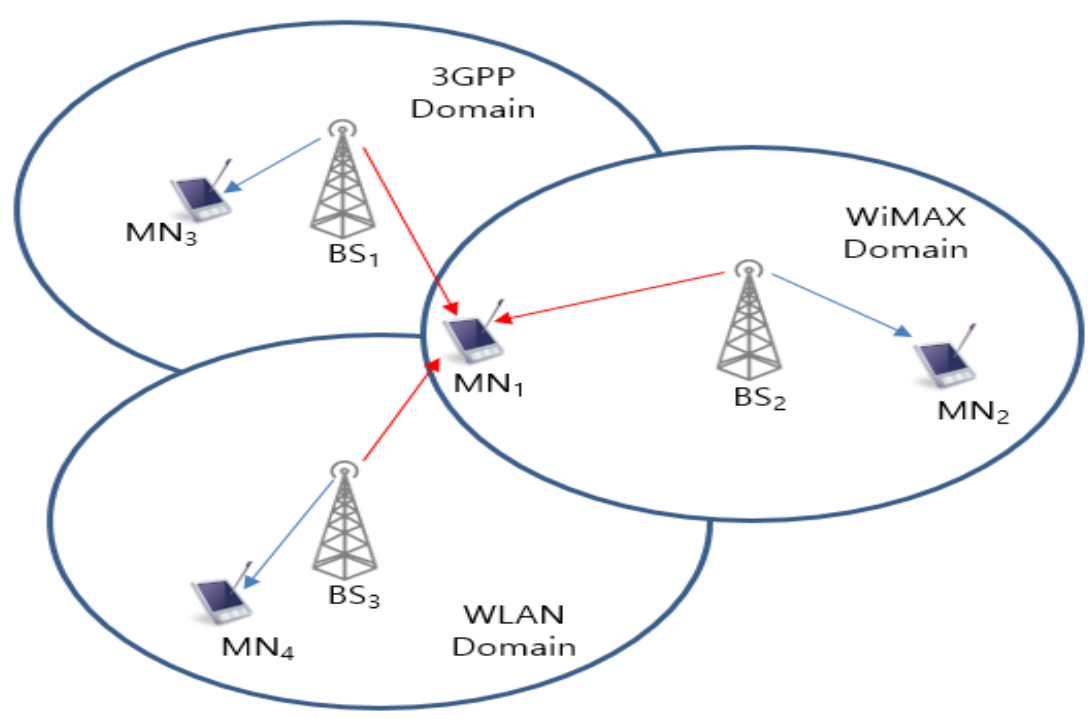

\section{Figure 4. Intercell Cooperation for Integrated Heterogeneous Wireless Networks}

The different access networks identified in Figure 4 can be represented by the media access gateways (MAGs) in order to control the different MNs that attaches to its access links. That is, MAG1 represents the WiMAX domain, MAG2 represents the WLAN domain, and MAG3 represents the 3GPP domain. Initially the $\mathrm{MN}_{1}$ is assumed to attach first to the WiMAX domain (MAG1) as indicated in Figure 5. The $\mathrm{MN}_{1}$ can always disconnect to MAG1 in the case that the network resources in MAG1 does not work for its needs. It can either attach to MAG2 or MAG3 depending on the identified network characteristics. These can be identified by the MN through the information received from MAG router advertisements. The router advertisement will include Flag bits indicating the current status of the access network. The Flag bits can determine the bandwidth, and the volume of data traffic that is being processed by the sending MAG. In this way, the tunnel to which the flow of multimedia contents will be forwarded can be set by the LMA to a best performing access network. 


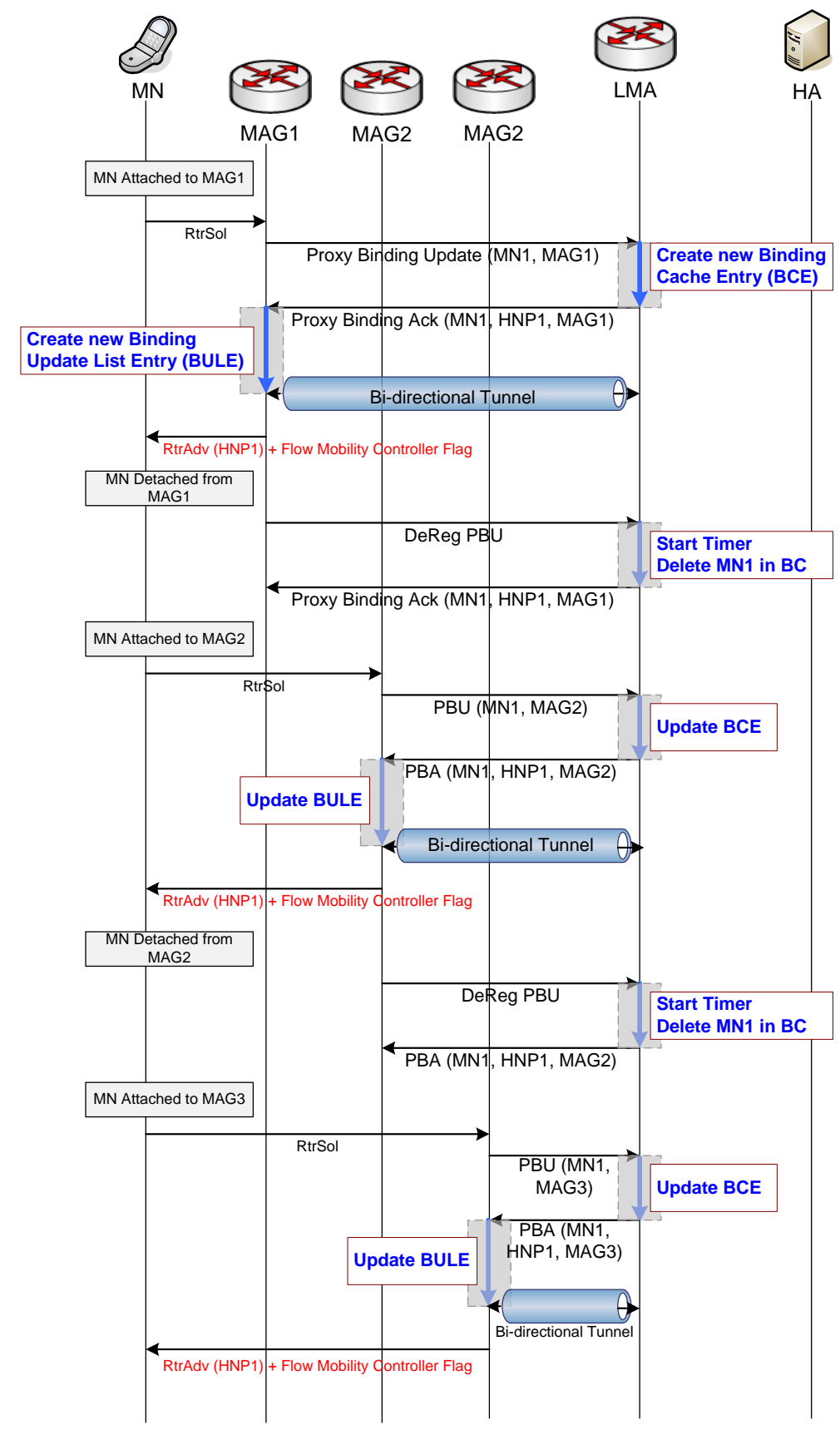

Figure 5. PMIPv6 Based Multi-MAC Mobility Management

The advantage of the multi-MAC flow mobility scheme is that the MN1 is enabled to switch between the available heterogeneous access networks without changing its IP address. That is, there will be no change for the MN's home network prefix (HNP) even if it is connected to MAG1, MAG2, or MAG3. In addition, the local mobility of mobile devices is managed by MAG through sending the updates to the LMA instead of the MN. The LMA in return just need to update its binding cache (BC) with the current MAG to which the MN is currently attached.

The network-based mobility management support of PMIPv6 and its extensions has become the focal point of to provide mobility support for integrated heterogeneous wireless networks. It allows mobility support without relying the mobility-related configurations to the mobile terminals. 


\section{Conclusion}

This paper has presented an analysis of a multi-MAC flow distribution management of multimedia contents across the integrated heterogeneous wireless networks. The MN's independence with the mobility-related signaling in PMIPv6 provides a mobility support that allows it to switch between the interrelated access networks. The MN's movement from one access network to another is based on the evaluated network conditions such as bandwidth, handoff latency, number of connected users, volume of data traffic being processed, etc. The multi-MAC flow mobility for mobile devices were utilized for the efficient and optimized distribution of multimedia data IP contents and a centralized control over the handoffs optimization between the available interrelated heterogeneous wireless networks. In the future, the interferences caused by overlapping access network technologies will be analyzed.

\section{Acknowledgments}

This research was supported by Basic Science Research Program through the National Research Foundation of Korea (NRF) funded by the Ministry of Science, ICT and future planning (2015R1A2A2A03002851).

\section{References}

[1] D. Johnson, C. Perkins, J. Arkko, "Mobility Support in IPv6", Internet Engineering Task Force (IETF), RFC 3775, (2004) June.

[2] C. Perkins, D. Johnson, J. Arkko, "Mobility Support in IPv6", Internet Engineering Task Force (IETF), RFC 6275, ISSN: 2070-1721, (2011) July.

[3] R. Koodli, "Fast handovers for mobile IPv6", Internet Engineering Task Force (IETF), RFC 4068, (2005) July.

[4] V. Visoottiviseth, P. Ngamtura, "On the Performance of MIPv6 and FMIPv6 based on Real IPv6 applications over IEEE 802.11g Testbeds", Int. Symposium on Commun. and Inf. Technol. (ISCIT), (2010) October, pp. 1217-1222.

[5] H. Soliman, C. Castelluccia, K. E. Malki, L. Bellier, "Hierarchical mobile IPv6 mobility management (HMIPv6)”, Internet Engineering Task Force (IETF), RFC 4140, (2005) August.

[6] H. Soliman, C. Castelluccia, K. ElMalki and L. Bellier, "Hierarchical Mobile IPv6 (HMIPv6) Mobility Management", Internet Engineering Task Force (IETF), RFC No. 5380, http://www.rfc-base.org/txt/rfc4140.txt, (2008) October.

[7] S. Gundavelli, K. Leung, V. Devarapalli, K. Chowdhury, B. Patil, "Proxy mobile IPv6", Internet Engineering Task Force (IETF), RFC 5213, (2008) August.

[8] C. J. Bernardos, M. Gramaglia, L. M. Contreras, M. Calderon, I. Soto, "Network-based Localized IP mobility Management: Proxy Mobile IPv6 and Current Trends in Standardization", Journal of Wireless Mobile Networks, Ubiquitous Computing, and Dependable Applications (JoWUA)(Special issue: Advances in Wireless Mobile and Sensor Technologies), vol. 1, no. 2/3, (2010), pp. 16-35. 
International Journal of Control and Automation Vol.10, No.4 (2017) 